\title{
Classification of healthcare-associated infection: a systematic review 10 years after the first proposal
}

Teresa Cardoso ${ }^{1 *}$, Mónica Almeida², N Deborah Friedman³ , Irene Aragão', Altamiro Costa-Pereira4, António E Sarmento ${ }^{5}$ and Luís Azevedo ${ }^{4}$

\begin{abstract}
Background: Ten years after the first proposal, a consensus definition of healthcare-associated infection (HCAl) has not been reached, preventing the development of specific treatment recommendations. A systematic review of all definitions of HCAl used in clinical studies is made.

Methods: The search strategy focused on an HCAI definition. MEDLINE, SCOPUS and ISI Web of Knowledge were searched for articles published from earliest achievable data until November 2012. Abstracts from scientific meetings were searched for relevant abstracts along with a manual search of references from reports, earlier reviews and retrieved studies.

Results: The search retrieved 49,405 references: 15,311 were duplicates and 33,828 were excluded based on title and abstract. Of the remaining 266,43 met the inclusion criteria. The definition more frequently used was the initial proposed in 2002 - an infection present at hospital admission or within 48 hours of admission in patients that fulfilled any of the following criteria: received intravenous therapy at home, wound care or specialized nursing care in the previous 30 days; attended a hospital or hemodialysis clinic or received intravenous chemotherapy in the previous 30 days; were hospitalized in an acute care hospital for $\geq 2$ days in the previous 90 days, resided in a nursing home or long-term care facility. Additional criteria founded in other studies were: immunosuppression, active or metastatic cancer, previous radiation therapy, transfer from another care facility, elderly or physically disabled persons who need healthcare, previous submission to invasive procedures, surgery performed in the last 180 days, family member with a multi-drug resistant microorganism and recent treatment with antibiotics.

Conclusions: Based on the evidence gathered we conclude that the definition initially proposed is widely accepted. In a future revision, recent invasive procedures, hospitalization in the last year or previous antibiotic treatment should be considered for inclusion in the definition. The role of immunosuppression in the definition of HCAl still requires ongoing discussion.
\end{abstract}

Keywords: Healthcare-associated infection, Classification, Multidrug resistant pathogens prevalence, Pneumonia, Bloodstream infections, Endocarditis, Urinary tract infections, Intra-abdominal infections

\section{Background}

Traditionally, infections have been classified as community or hospital-acquired, according to their place of acquisition, and this classification is still used to guide treatment decisions $[1,2]$.

Over the last decade the massive increase in outpatient clinical care has led to a new context for the emergence

\footnotetext{
* Correspondence: cardoso.tmc@gmail.com

'Intensive Care Unit, Unidade de Cuidados Intensivos Polivalente, Hospital de Santo António, University of Porto, Largo Prof. Abel Salazar, 4099-001 Porto, Portugal

Full list of author information is available at the end of the article
}

of healthcare-associated infections (HCAI). This is a new name for a new group of infections emerging among patients that come from the community with a history of previous exposure to healthcare who do not fit the nosocomial infection criteria. The proportion of patients hospitalized with HCAI among those admitted from the community setting can be as high as 50\% [3-6].

The first proposals of HCAI and its inclusion in infection classification along with community-acquired infection (CAI) and hospital-acquired infection (HAI) were made in 2002 by Siegman-Igra et al. [7] and Friedman et al. 
[3]. Different one from another, the definition from Friedman et al. [3] has been used in numerous clinical studies and will be referred to in this review as the initial definition; it is defined as an infection present at hospital admission or within 48 hours of admission in patients that fulfilled any of the following criteria:

- received intravenous therapy at home, wound care or specialized nursing care through a healthcare agency, family or friends; or had self-administered intravenous medical therapy in the 30 days before the infection;

- attended a hospital or hemodialysis clinic or received intravenous chemotherapy in the previous 30 days;

- were hospitalized in an acute care hospital for 2 or more days in the previous 90 days,

- resided in a nursing home or long-term care facility.

Although widely accepted [5,8-10] numerous alternative definitions have also been used in clinical studies [11-14]. This heterogeneity has raised more confusion than understanding in determining likely microbiological resistance patterns and making decisions about empiric antibiotic treatment. A correct recognition of all risk factors for $\mathrm{HCAI}$ is crucial in guaranteeing optimal empiric antibiotic choice to adequately treat likely pathogens while avoiding selective pressure that contributes to the development of multidrug-resistant (MDR) organisms.

The objective of the current study is to present a systematic review of all definitions of HCAI used in clinical studies in order to compare and contrast the criteria they include.

\section{Methods}

\section{Data sources and searches}

This search was performed in accordance with the recommendations of the Cochrane collaboration using MEDLINE/PubMed, SCOPUS and ISI Web of Knowledge from the earliest achievable data until November 2012. A manual search of references from reports, earlier reviews and retrieved studies was also performed. Abstract books and CD-ROMs from several annual scientific meetings were searched for relevant abstracts (Figure 1). No language restriction was applied and papers written in a foreign language were translated.

The electronic search strategy covered the main subject area: healthcare-associated infection (Additional file 1: Search strategy details). The last search was done on 8 November 2012.

\section{Study selection}

The inclusion criteria were all observational studies (cohort, cross-sectional or case-control) on adult patients admitted to hospital that provided microbiology results according to place of acquisition of infection. The following definitions of infection by setting were used:

- CAI - infection detected within 48 hours of hospital admission in patients without previous contact with healthcare service.

- HAI - localized or systemic condition: 1) that results from adverse reaction to the presence of an infectious agent(s) or its toxin(s) and 2) that was present 48 hours or more after hospital admission and not incubating at hospital admission time [15].

- HCAI - infection detected within 48 hours of hospital admission in patients that had previous contact with healthcare service within one year.

\section{Data extraction and quality assessment}

The results of the literature search were accessed by two reviewers (TC, MA) and non-relevant studies were excluded based on title and abstract. For potentially relevant studies, the full text was obtained, and two investigators (TC, MA) independently assessed study eligibility and extracted data on study design, objectives, HCAI definitions and multi-drug resistant pathogens (MDR) prevalence (Methicillin-resistant Staphylococcus aureus (MRSA), Pseudomonas aeruginosa, Acinectobacter, Stenotrophomas maltophilia, extended-spectrum beta lactamases producer (ESBL)), using a data extraction protocol; disagreements were resolved through consultation with a third reviewer (LA).

Each selected study was independently evaluated by two reviewers (TC, MA) for the strength of evidence through examination of the study design and quality of data.

Potential threats to the internal validity of included studies were evaluated considering the following criteria:

- The authors define inclusion criteria,

- The authors define an adequate selection method,

- The selection of participants was consecutive,

- The outcome data (microbiology data by place of acquisition) were complete and reported (no attrition bias) and

- All results were reported (reporting bias).

Studies that met all of the above five criteria, were classified as "low risk of bias". Studies that partially met one or more criteria were classified as "moderate risk of bias". Studies were classified as "high risk of bias" if one or more of these criteria was not met.

\section{Data analysis}

Data on individual studies included are provided in Tables 1 and 2. A meta-analysis was not performed due to the nature of the objectives of this review and the heterogeneity of the studies included. 


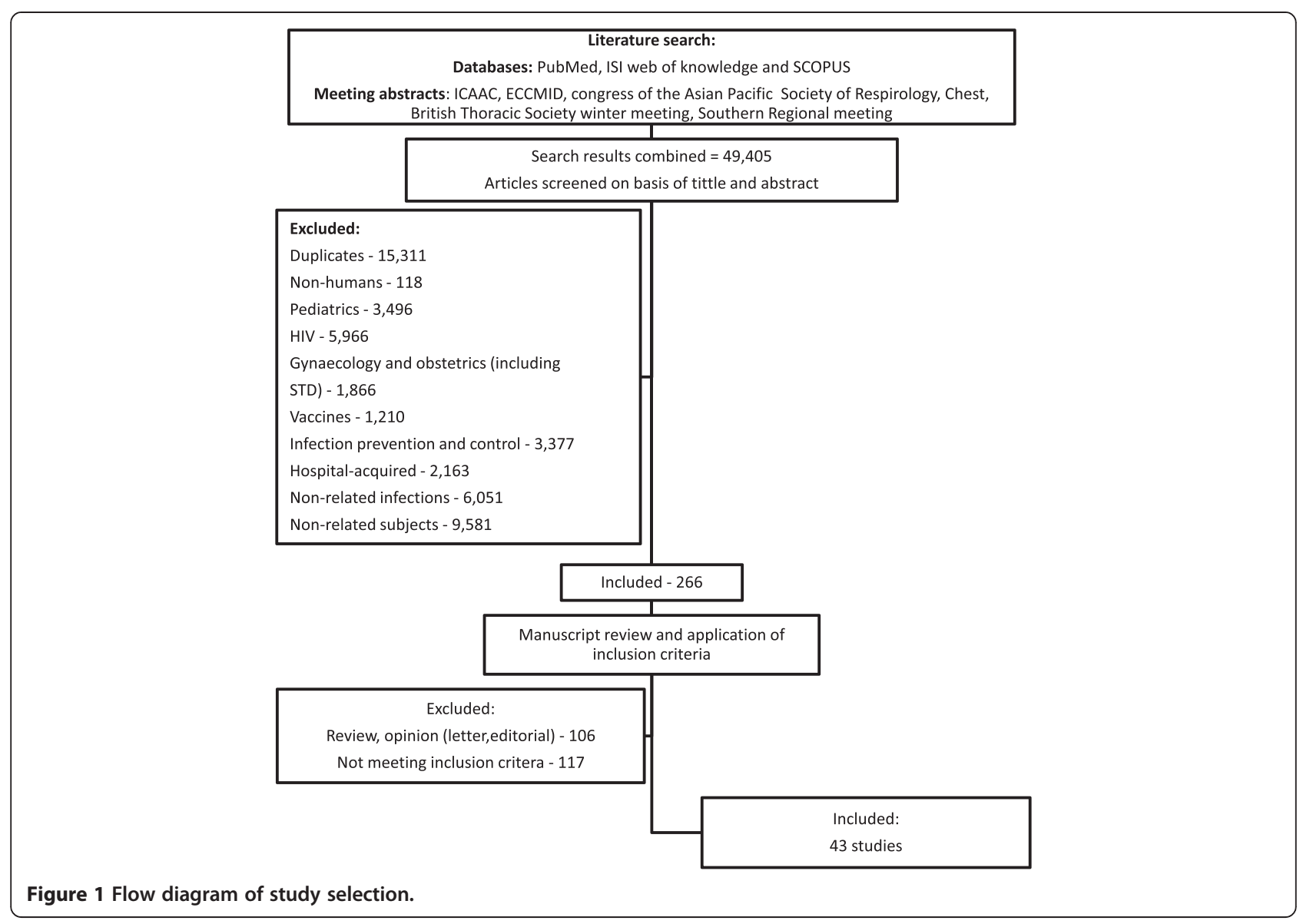

\section{Results}

The search retrieved a total of 49,405 references. Of the 266 studies included in the first review, 106 were review articles or opinion pieces and 117 did not meet inclusion criteria. Of the remaining 52 studies: 30 used the initial definition of Friedman et al. [3], but only 21 provided data on microbiology and were included along with 22 additional studies that used alternative definitions and met the inclusion criteria. Of the 43 studies included in this systematic review (Figure 1): 18 were prospective (7 multicenter and 11 single center) and 25 were retrospective ( 9 multicenter and 16 single center); involving 42,611 patients.

Characteristics of included studies that used the initial definition are shown in Table 1 and of those that used alternative definitions in Table 2.

\section{Infections by source}

In bloodstream HCAIs, six studies used the initial definition [3] (Table 1) and six did not (Table 2), all found an increasing prevalence of MDR organisms from CAI to HCAI and HAI, regardless of the definition used.

The majority of the included studies were about pneumonia (24 studies of 43). Most of these studies only compared community-acquired pneumonia (CAP) with healthcare-associated pneumonia (HCAP) and revealed a higher prevalence of MDR pathogens among HCAP patients compared with CAP patients. There were three studies comparing CAP and HCAP with hospital acquired pneumonia (HAP) $[13,21,42]$ but they used different definitions of HCAP achieving different results regarding MDR prevalence according to place of acquisition of infection (Tables 1 and 2).

There were three studies of healthcare-associated infective endocarditis $[5,29,46]$. Two found an increasing rate of MDR organisms from community-acquired to healthcareassociated and hospital-acquired infective endocarditis (Table 1). A third study compared healthcare-associated infective endocarditis with non-healthcare-associated infective endocarditis (that is, community-acquired plus hospital-acquired infective endocarditis) and found a higher prevalence of MDR in healthcare-associated infective endocarditis than in non-healthcare-associated infective endocarditis (Table 2).

Studies regarding urinary tract $[6,45]$ and intra-abdominal [43] infections also found a higher prevalence of MDR organisms, among HCAIs when compared to CAIs (Tables 1 and 2). 
Table 1 Characterization of included studies that use the initial definition of HCAI [3] by focus of infection

\begin{tabular}{|c|c|c|c|c|c|}
\hline Author, publication year & Study design & $\mathrm{MDR}^{*}$ & organisms & , n (\%) & Risk of bias \\
\hline Bacteriemia & & CAI & HCAI & HAI & \\
\hline Friedman et al., 2002 [3] & Prospective, multicenter, 504 patients, USA, 4 to 5 months & & Not mention & & High \\
\hline Marschall et al., 2009 [16] & Prospective, single center, 250 patients, Gram-negative, USA, 6 months & $0(0)$ & $9(7)$ & $10(11)$ & Low \\
\hline Evans et al., 2009 [17] & Retrospective, multicenter, 223 patients, spinal cord injury, USA, 7 years & $6(17)$ & $34(31)$ & $111(42)$ & High \\
\hline Son et al., 2010 [18] & Prospective, multicenter, 1,144 patients, Korea, 12 months & $29(8)$ & $38(7)$ & $162(79)$ & Low \\
\hline $\begin{array}{l}\text { Rodriguez-Bano et al., } \\
2010 \text { [19] }\end{array}$ & $\begin{array}{l}\text { Prospective, multicenter, } 821 \text { bacteremia episodes including potential } \\
\text { contaminants, Spain, } 2 \text { to } 5 \text { months }\end{array}$ & $7(5)$ & $29(15)$ & $99(21)$ & Moderate \\
\hline Vallés et al., 2011 [8] & Prospective, multicenter, 726 patients, Spain and Argentina, 12 months & $7(2)$ & $11(8)$ & $29(12)$ & High \\
\hline Pneumonia & & CAI & HCAI & & \\
\hline Carratalà et al., 2007 [10] & $\begin{array}{l}\text { Prospective, single center, } 727 \text { patients, those with neutropenia, AIDS } \\
\text { and after transplantation were excluded, Spain, } 4 \text { years. }\end{array}$ & $3(1)$ & $2(2)$ & & Low \\
\hline Shindo et al., 2009 [20] & Retrospective, single center, 371 patients, Japan, 1 year and 3 months & $6(6)$ & $17(22)$ & & Low \\
\hline Park et al., 2010 [9] & $\begin{array}{l}\text { Retrospective, single center, } 345 \text { patients, CAI and HCAI were considered } \\
\text { until } 72 \mathrm{~h} \text { after hospital admission; patients with neutropenia, AIDS and } \\
\text { after transplantation were excluded, Korea, } 1 \text { year. }\end{array}$ & $7(15)$ & $21(32)$ & & Moderate \\
\hline Pascual et al., 2010 [21] & $\begin{array}{l}\text { Retrospective, single center, } 308 \text { patients with bacteriemic pneumonia, } \\
\text { Spain, } 6 \text { years }\end{array}$ & CAI (2) & HCAl (12) & $\mathrm{HAl}(31)$ & High \\
\hline Umeki et al., 2011 [22] & Prospective, single center, 202 patients, Japan, 2 years & $10(21)$ & $12(25)$ & & Moderate \\
\hline Seki et al., 2011 [23] & Retrospective, single center, 34 patients Japan, 4 months & $0(0)$ & $6(43)$ & & Moderate \\
\hline Garcia-Vidal et al., 2011 [24] & $\begin{array}{l}\text { Prospective, single center, 2,153 patients, those with more than one } \\
\text { condition of HCAl, with neutropenia, AIDS, after transplantation and chronic } \\
\text { corticosteroid treatment were excluded, Spain, } 8 \text { years and } 9 \text { months }\end{array}$ & $19(2)$ & $7(2)$ & & Low \\
\hline Jung et al., 2011 [25] & Retrospective, single center, 527 patients, Korea, 1 year & $15(18)$ & $30(38)$ & & Low \\
\hline Jeon et al., 2011 [26] & $\begin{array}{l}\text { Retrospective, multicenter, } 210 \text { patients older than } 60 \text { years, Korea, } \\
2 \text { years. }\end{array}$ & $10(16)$ & $20(67)$ & & Low \\
\hline Depuydt et al. 2011 [27] & $\begin{array}{l}\text { Retrospective, single center, } 269 \text { patients, those with neutropenia, } \\
\text { transplantation or transferred from another hospital were excluded, } \\
\text { Belgium, } 1 \text { year }\end{array}$ & $0(0)$ & $6(30)$ & & Low \\
\hline Park et al., 2012 [4] & Prospective, single center, 339 patients, Korea, 2 years & $35(20)$ & $52(31)$ & & Low \\
\hline Lee et al., 2012 [28] & Retrospective, multicenter study, 250 patients, Korea, 21 months & $3(4)$ & $17(31)$ & & Low \\
\hline Other foci & & CAI & HCAI & HAI & \\
\hline Benito et al., 2009 [5] & $\begin{array}{l}\text { Prospective, single center, } 1622 \text { patients with endocarditis, comparing } \\
\text { CAI with healthcare associated that included non-nosocomial and } \\
\text { nosocomial, intra-venous drug users and prosthetic valves were } \\
\text { excluded, USA, } 6 \text { months. }\end{array}$ & $25(3)$ & $41(17)$ & $76(26)$ & Low \\
\hline Wu et al., 2011 [29] & Retrospective, single center,192 patients with endocarditis, Taiwan, 5 years & $15(11)$ & $13(43)$ & $17(81)$ & Moderate \\
\hline Aguilar-Duran et al., 2012 [6] & $\begin{array}{l}\text { Prospective, single center, } 251 \text { patients with urinary infection, Spain, } \\
7 \text { to } 8 \text { months }\end{array}$ & $2(2)$ & $15(15)$ & $9(14)$ & High \\
\hline
\end{tabular}

*MDR, MRSA, Pseudomonas sp, Acinectobacter, Stenotrophomonas maltophilia, ESBL. CAl, community-acquired infection; HAI, hospital-acquired infection; HCAl, healthcare-associated infection; MDR, multidrug-resistant.

The initial definition [3] was the most widely used in clinical studies (30 studies among 52). Overall, different HCAI definitions comprised 17 different criteria, of which 7 were equivalent to those used by Friedman et al. [3], but leading to a different final definition due to the addition or subtraction of criteria (Table 3).

An analysis of the risk of bias of the 43 included studies revealed that 24 presented a low risk of bias, 7 presented a moderate risk of bias, and 12 presented a high risk of bias, according to previously defined criteria (Additional file 2:
eTable 1 - Studies with moderate or high risk of bias according to pre-defined criteria).

\section{Discussion}

Ten years after the first descriptions [3,7], this is the first systematic review of HCAI classification. It incorporates all published studies on HCAI that provided original data. The majority of the included studies had a low risk of bias, resulting in good quality of the evidence assembled. 
Table 2 Characterization of included studies that did not use the initial definition of HCAI [3] by focus of infection Study, year Study design HCAI criteria MDR* organisms Risk of bias of publication

\section{A. Bloodstream infections}

Siegman-lgra et al., Prospective, single center, 1,028 2002 [7] infections in 912 patients; Israel, 1 year

Shorr et al., 2006 [11]

Retrospective, multicenter, 6,697 patients; USA, 2 years

Kao et al., 2011 [30]

Kollef et al., 2011 [12]

Al-Hasan et al., 2012 [31]

Lenz et al., 2012 [32]
Prospective, multicenter, 1,143 patients; USA, 1 year Retrospective, multicenter, 733

Prospective, single center, 890 infections in 831 patients older than 14 years; Taiwan, 1 year episodes of gram negative bacteremia; excludes polymicrobial, nosocomial and recurrent episodes; USA, 10 years

Retrospective, multicenter, 7,712 patients; only samples obtained in the first 5 days of hospital admission; Canada, 8 years
1. Discharge from hospital 2 to 30 days previously

2. Nursing-home acquired

3. Patients with long-term intravenous devices, for hemodialysis, chemotherapy or parenteral nutrition

4. Chronic hemodialysis

5. Invasive procedure previously or at hospital admission

1. Prior hospitalization within 30 days

2. Transfer from another healthcare facility

3. Chronic hemodialysis

4. Immunosuppression medication or metastatic cancer

1. Hospitalized for 2 or more days in the previous 90 days

\section{Resided in a nursing home}

3. Hemodialysis, intravenous chemotherapy or invasive procedures in the previous 90 days

1. Prior hospitalization within 6 months

2. Admission from a skilled nursing facility

3. Hemodialysis

4. Immunosuppression

1. Hospitalized for 2 or more days in the previous 90 days

2. Residents in a nursing home or long-term care facility

3. Received intravenous therapy including ATB and chemotherapy

4. Hemodialysis in the previous 30 days

1. Hospitalized for 2 or more days in the previous 90 days

2. Nursing home or long term-care facility department within the prior 5 to 30 days

4. Hemodialysis

5. Active cancer

CAl HCAl HAl Moderate

$8(2) \quad 24(10) \quad 71(17)$

CAl HCAl HAl LOW

$152(2) \quad 397(11) \quad 62(13)$

CAl HCAl HAl High

$11(2) \quad 41(16) \quad 42(41)$

CAI HCAI LOW

79 (19) $242(33)$

CAl HCAl HAl LOW 
Table 2 Characterization of included studies that did not use the initial definition of HCAI [3] by focus of infection (Continued)

\begin{tabular}{|c|c|c|c|c|c|}
\hline \multicolumn{6}{|l|}{ B. Pneumonia } \\
\hline \multirow[t]{3}{*}{$\begin{array}{l}\text { Kollef et al., } \\
2006[13]\end{array}$} & $\begin{array}{l}\text { Retrospective, multicenter, } \\
4,543 \text { patients with positive } \\
\text { cultures within } 5 \text { days of } \\
\text { hospital admission, USA, } \\
2 \text { years }\end{array}$ & 1. Prior hospitalization within 30 days & CAl & $\mathrm{HCAl}$ & High \\
\hline & & 2. Admission from another care facility & $624(28)$ & $537(54) \quad 362(43)$ & \\
\hline & & 3. Receiving long-term hemodialysis & & & \\
\hline \multirow[t]{4}{*}{$\begin{array}{l}\text { Micek et al., } \\
2007 \text { [33] }\end{array}$} & $\begin{array}{l}\text { Retrospective, single center, } 639 \\
\text { patients older than } 16 \text { years with } \\
\text { positive cultures, Spain, } 2 \text { years }\end{array}$ & 1. Hospitalization in the past 12 months & CAl & $\mathrm{HCAl}$ & Low \\
\hline & & $\begin{array}{l}\text { 2. Resident in a nursing home or long-term } \\
\text { care facility or rehabilitation hospital }\end{array}$ & $35(17)$ & $242(56)$ & \\
\hline & & $\begin{array}{l}\text { 3. Outpatient hemodialysis, peritoneal } \\
\text { dialysis or infusion therapies requiring } \\
\text { regular visits to a clinic }\end{array}$ & & & \\
\hline & & 4. Immunosuppression & & & \\
\hline \multirow[t]{6}{*}{$\begin{array}{l}\text { Schreiber et al., } \\
2010 \text { [34] }\end{array}$} & $\begin{array}{l}\text { Retrospective, single center study, } \\
190 \text { patients needing mechanical } \\
\text { ventilation more than } 24 \text { hours after } \\
\text { hospital admission, patients without } \\
\text { evidence of bacterial infection and } \\
\text { patients transferred from other } \\
\text { hospitals were excluded, USA, } \\
4 \text { years }\end{array}$ & 1. Recent hospitalization (90 days) & CAl & $\mathrm{HCAl}$ & Low \\
\hline & & 2. Admission from a long-term facility & $17(18)$ & $48(48)$ & \\
\hline & & $\begin{array}{l}\text { 3. Recent treatment with broad spectrum } \\
\text { antibiotics ( } 30 \text { days) }\end{array}$ & & & \\
\hline & & 4. Chronic hemodialysis & & & \\
\hline & & 5. Wound care & & & \\
\hline & & 6. Immunosuppression & & & \\
\hline \multirow[t]{4}{*}{$\begin{array}{l}\text { Grenier et al., } \\
2011[14]\end{array}$} & $\begin{array}{l}\text { Retrospective, single center study, } \\
3,295 \text { patients, those transferred from } \\
\text { other hospitals or discharged from an } \\
\text { acute care facility within } 14 \text { days were } \\
\text { excluded, Canada, } 12 \text { years }\end{array}$ & $\begin{array}{l}\text { 1. Hospitalization in the past } 90 \text { days, but } \\
\text { not in the last } 14 \text { days }\end{array}$ & CAl & $\mathrm{HCAl}$ & Low \\
\hline & & $\begin{array}{l}\text { 2. Resident of a nursing home or } \\
\text { long-term care facility }\end{array}$ & $42(4)$ & $21(10)$ & \\
\hline & & $\begin{array}{l}\text { 3. Outpatient intravenous therapy or } \\
\text { cancer therapy within the previous month }\end{array}$ & & & \\
\hline & & 4. Long term hemodialysis & & & \\
\hline \multirow[t]{6}{*}{$\begin{array}{l}\text { Guimarães et al., } \\
2011 \text { [35] }\end{array}$} & $\begin{array}{l}\text { Retrospective, single center study, } \\
197 \text { patients, Portugal, } 1 \text { year }\end{array}$ & $\begin{array}{l}\text { 1. Hospitalization for } 2 \text { days or more in the } \\
\text { preceding } 90 \text { days }\end{array}$ & CAl & $\mathrm{HCAl}$ & High \\
\hline & & $\begin{array}{l}\text { 2. Resident of a nursing home or } \\
\text { extended-care facility }\end{array}$ & $2(18)$ & $9(82)$ & \\
\hline & & $\begin{array}{l}\text { 3. Intravenous antibiotic therapy or } \\
\text { chemotherapy within } 30 \text { days }\end{array}$ & & & \\
\hline & & 4. Chronic dialysis within 30 days & & & \\
\hline & & 5. Home wound care & & & \\
\hline & & $\begin{array}{l}\text { 6. Family member with multi-drug } \\
\text { resistant pathogen }\end{array}$ & & & \\
\hline
\end{tabular}


Table 2 Characterization of included studies that did not use the initial definition of HCAI [3] by focus of infection (Continued)

\begin{tabular}{|c|c|c|c|c|c|}
\hline \multirow[t]{6}{*}{$\begin{array}{l}\text { Chalmers et al., } \\
2011[36]\end{array}$} & $\begin{array}{l}\text { Prospective, single center, 1,348 } \\
\text { patients; Scotland, } 4 \text { years and } \\
5 \text { months }\end{array}$ & $\begin{array}{l}\text { 1. Hospitalization for } 2 \text { days or more in the } \\
\text { preceding } 90 \text { days }\end{array}$ & CAl & HCAl & High \\
\hline & & $\begin{array}{l}\text { 2. Resident of a nursing home or } \\
\text { extended-care facility }\end{array}$ & $3(1)$ & $5(6)$ & \\
\hline & & $\begin{array}{l}\text { 3. Home infusion therapy (including } \\
\text { antibiotics and long-indwelling devices } \\
\text { as catheters) }\end{array}$ & & & \\
\hline & & 4. Chronic dialysis within 30 days & & & \\
\hline & & 5. Home wound care & & & \\
\hline & & $\begin{array}{l}\text { 6. Family member with multi-drug resistant } \\
\text { pathogen }\end{array}$ & & & \\
\hline \multirow[t]{6}{*}{$\begin{array}{l}\text { Tasbakan et al., } \\
2011[37]\end{array}$} & $\begin{array}{l}\text { Retrospective, single center study, } \\
187 \text { patients, Turkey, } 1 \text { year }\end{array}$ & $\begin{array}{l}\text { 1. Hospitalization for } 2 \text { days or more in the } \\
\text { preceding } 90 \text { days }\end{array}$ & CAl & HCAl & Low \\
\hline & & $\begin{array}{l}\text { 2. Resident of a nursing home or } \\
\text { extended-care facility }\end{array}$ & $1(13)$ & $17(44)$ & \\
\hline & & $\begin{array}{l}\text { 3. Home infusion therapy } \\
\text { (including antibiotics) }\end{array}$ & & & \\
\hline & & 4. Chronic dialysis within 30 days & & & \\
\hline & & 5. Home wound care & & & \\
\hline & & $\begin{array}{l}\text { 6. Family member with multi-drug resistant } \\
\text { pathogen }\end{array}$ & & & \\
\hline \multirow[t]{4}{*}{$\begin{array}{l}\text { Ishida et al., } \\
2012 \text { [38] }\end{array}$} & $\begin{array}{l}\text { Retrospective, single center, } 893 \\
\text { patients; Japan, } 3 \text { years }\end{array}$ & $\begin{array}{l}\text { 1. Discharged from a hospital in the } \\
\text { preceding } 90 \text { days }\end{array}$ & CAl & $\mathrm{HCAl}$ & Low \\
\hline & & $\begin{array}{l}\text { 2. Resident in a nursing home or } \\
\text { extended-care ward }\end{array}$ & $6(3)$ & $37(21)$ & \\
\hline & & $\begin{array}{l}\text { 3. A patient who regularly requires vascular } \\
\text { access for dialysis, antimicrobial treatment, } \\
\text { chemotherapy or immunosuppressive } \\
\text { therapy in an outpatient setting }\end{array}$ & & & \\
\hline & & $\begin{array}{l}\text { 4. Elderly or handicap who needs } \\
\text { long-term care with an ECOG of } 3 \text { or } 4\end{array}$ & & & \\
\hline \multirow[t]{4}{*}{$\begin{array}{l}\text { Miyashita et al., } \\
2012 \text { [39] }\end{array}$} & $\begin{array}{l}\text { Prospective, multicenter, 1,385 } \\
\text { patients; CAl and HCAI were } \\
\text { considered until } 72 \mathrm{~h} \text { after } \\
\text { hospital admission, Japan, } 6 \text { years }\end{array}$ & $\begin{array}{l}\text { 1. Discharged from a hospital in the } \\
\text { preceding } 90 \text { days }\end{array}$ & CAl & HCAl & High \\
\hline & & $\begin{array}{l}\text { 2. Resident in a long-term nursing home } \\
\text { setting or healthcare home }\end{array}$ & $21(4)$ & $78(20)$ & \\
\hline & & $\begin{array}{l}\text { 3. Continuous receiving endovascular } \\
\text { therapy in an ambulatory setting (including } \\
\text { dialysis, antibiotics, anticancer drugs and } \\
\text { immunosuppression) }\end{array}$ & & & \\
\hline & & $\begin{array}{l}\text { 4. Elderly persons or physical disable } \\
\text { persons who need healthcare }\end{array}$ & & & \\
\hline \multirow[t]{2}{*}{$\begin{array}{l}\text { Wu et al., } \\
2012[40]\end{array}$} & $\begin{array}{l}\text { Retrospective, multicenter study, } \\
1,646 \text { patients, those with pneumonia } \\
\text { developing } 2 \text { days after admission or } \\
\text { less than } 14 \text { days after the last } \\
\text { hospitalization, with lung cancer } \\
\text { obstructive pneumonia or HIV } \\
\text { positive status with CD4+ }<<200 \\
\text { were excluded, Taiwan, } 1 \text { year }\end{array}$ & $\begin{array}{l}\text { 1. Undergoing repeated hospitalization } \\
\text { within } 90 \text { days before }\end{array}$ & CAl & HCAl & Low \\
\hline & & 2. Residing in a nursing home & $122(15)$ & $169(28)$ & \\
\hline
\end{tabular}


Table 2 Characterization of included studies that did not use the initial definition of HCAI [3] by focus of infection (Continued)

\begin{tabular}{|c|c|c|c|c|c|c|}
\hline & & $\begin{array}{l}\text { 3. Receiving radiation therapy or } \\
\text { chemotherapy at an outpatient clinic }\end{array}$ & & & & \\
\hline & & $\begin{array}{l}\text { 4. Received regular dialysis at an out } \\
\text { patients clinic }\end{array}$ & & & & \\
\hline $\begin{array}{l}\text { Sugisaki et al., } \\
2012[41]\end{array}$ & $\begin{array}{l}\text { Retrospective, single center } \\
\text { study, } 526 \text { patients, Japan, } \\
4 \text { years }\end{array}$ & $\begin{array}{l}\text { 1. Hospitalization for } 2 \text { days or more in the } \\
\text { preceding } 90 \text { days }\end{array}$ & CAl & & CAl & Low \\
\hline & & $\begin{array}{l}\text { 2. Resident of a nursing home or } \\
\text { extended-care facility }\end{array}$ & $50(15)$ & & (40) & \\
\hline & & $\begin{array}{l}\text { 3. Home infusion therapy (including } \\
\text { antibiotics and long indwelling devices } \\
\text { as catheters) }\end{array}$ & & & & \\
\hline & & 4. Chronic dialysis within 30 days & & & & \\
\hline & & 5. Home wound care & & & & \\
\hline & & $\begin{array}{l}\text { 6. Family member with multi-drug } \\
\text { resistant pathogen }\end{array}$ & & & & \\
\hline $\begin{array}{l}\text { Giannella et al., } \\
2012[42]\end{array}$ & $\begin{array}{l}\text { Prospective, multicenter, 1,002 } \\
\text { patients older than } 16 \text { years } \\
\text { admitted into internal medicine } \\
\text { departments, Spain, } 2 \text { weeks }\end{array}$ & 1. Hospitalization in the past 180 days & CAl & $\mathrm{HCAl}$ & $\mathrm{HAl}$ & Low \\
\hline & & $\begin{array}{l}\text { 2. Resident in a nursing home or } \\
\text { extended-care facility }\end{array}$ & $6(4)$ & $19(29)$ & $11(52)$ & \\
\hline & & $\begin{array}{l}\text { 3. Attending a hospital regularly because } \\
\text { of chronic underlying disease }\end{array}$ & & & & \\
\hline & & 4. Undergoing hemodialysis & & & & \\
\hline & & $\begin{array}{l}\text { 5. Wound care or specialized nursing care } \\
\text { in the past } 30 \text { days }\end{array}$ & & & & \\
\hline & & 6. Chemotherapy in the past 30 days & & & & \\
\hline & & 7. Surgery in the past 180 days & & & & \\
\hline C. Other foci & & & & & & \\
\hline $\begin{array}{l}\text { Swenson et al., } \\
2009[43]\end{array}$ & $\begin{array}{l}\text { Retrospective, single center study, } \\
2,049 \text { intra-abdominal infections; } \\
\text { Canada, } 10 \text { year }\end{array}$ & $\begin{array}{l}\text { 1. Patients with a story of any } \\
\text { hospitalization in the previous } 30 \text { days }\end{array}$ & CAl & $\mathrm{HCAl}$ & & High \\
\hline & & $\begin{array}{l}\text { 2. Resident of a nursing home or } \\
\text { rehabilitation facility in the previous } 30 \text { days }\end{array}$ & $27(7)$ & & (28) & \\
\hline $\begin{array}{l}\text { Merli et al., } \\
2010 \text { [44] }\end{array}$ & $\begin{array}{l}\text { Prospective, single center study, } \\
\text { in } 54 \text { patients with cirrhosis, } \\
\text { patients with HIV infection, under } \\
\text { high dose of corticosteroid } \\
\text { treatment or immunosuppressive } \\
\text { therapy were excluded, Italy, } \\
9 \text { months }\end{array}$ & $\begin{array}{l}\text { 1. Hospitalization for } 2 \text { or more days or } \\
\text { had undergone surgery during the } \\
\text { preceding } 180 \text { days }\end{array}$ & CAl & $\mathrm{HCAl}$ & $\mathrm{HAl}$ & Low \\
\hline & & $\begin{array}{l}\text { 2. Resident of a nursing home or } \\
\text { long-term care facility }\end{array}$ & $2(50)$ & $9(82)$ & $5(45)$ & \\
\hline & & $\begin{array}{l}\text { 3. Attended a hospital or hemodialysis } \\
\text { clinic or received intravenous } \\
\text { chemotherapy in the } 30 \text { days before }\end{array}$ & & & & \\
\hline $\begin{array}{l}\text { Ha et al., } \\
2011[45]\end{array}$ & $\begin{array}{l}\text { Retrospective, single center study, in } \\
319 \text { patients with urinary infection; } \\
\text { Korea, } 1 \text { year }\end{array}$ & $\begin{array}{l}\text { 1. Hospitalization for } 2 \text { or more days in an } \\
\text { acute care hospital in the preceding } 90 \text { days }\end{array}$ & CAl & $\mathrm{HCAl}$ & & Low \\
\hline & & $\begin{array}{l}\text { 2. Resident of a nursing home or } \\
\text { long-term care facility }\end{array}$ & $1(1)$ & & (14) & \\
\hline
\end{tabular}




\begin{tabular}{|c|c|c|c|c|c|}
\hline & & $\begin{array}{l}\text { 3. Received intravenous therapy, wound } \\
\text { care or specialized nursing care at home } \\
\text { in the previous } 30 \text { days }\end{array}$ & & & \\
\hline & & $\begin{array}{l}\text { 4. Attended a hospital or hemodialysis } \\
\text { clinic or received intravenous } \\
\text { chemotherapy in the } 30 \text { days before }\end{array}$ & & & \\
\hline & & $\begin{array}{l}\text { 5. Received an invasive procedure, } \\
\text { urological surgery or urethral } \\
\text { catheterization in the previous } 7 \text { days }\end{array}$ & & & \\
\hline \multirow[t]{3}{*}{$\begin{array}{l}\text { Sy et al., } \\
2012[46]\end{array}$} & $\begin{array}{l}\text { Retrospective, multicenter study, } \\
\text { in } 1,536 \text { patients with endocarditis; } \\
\text { patients transferred from another } \\
\text { hospital, recurrent admissions or } \\
\text { day-stay admissions were excluded, } \\
\text { Australia, } 6 \text { years }\end{array}$ & $\begin{array}{l}\text { 1. Hospitalization for } 2 \text { days or more in the } \\
\text { preceding } 90 \text { days }\end{array}$ & $\mathrm{HCAl}$ & Non-HCAI & High \\
\hline & & $\begin{array}{l}\text { 2. Resident of a nursing home or in a } \\
\text { long-term facility }\end{array}$ & $40(26)$ & $61(18)$ & \\
\hline & & $\begin{array}{l}\text { 3. Attended a hemodialysis clinic or } \\
\text { received intravenous therapy in the } \\
30 \text { days before }\end{array}$ & & & \\
\hline
\end{tabular}

${ }^{*} \mathrm{MDR}=\mathrm{MRSA}$, Pseudomonas sp, Acinectobacter, Stenotrophomonas maltophilia, ESBL. CAl, community-acquired infection; ECOG, electrocochleography; HAl, hospital-acquired infection; HCAl, healthcare-associated infection; MDR, multidrug-resistant.

Table 3 List of all different criteria used to compose different classifications of HCAl

\begin{tabular}{|c|c|c|c|c|}
\hline Criteria for HCAl & Low risk of bias & Moderate risk of bias & High risk of bias & All studies \\
\hline Criteria included in the initial definition [3] & \multicolumn{4}{|c|}{ Number of studies (number of patients) } \\
\hline Received intravenous therapy & $16(13,082)$ & $7(3,239)$ & $8(6,284)$ & $31(22,605)$ \\
\hline Received wound care or specialized nursing care & $16(10,086)$ & $5(1,594)$ & $7(3,560)$ & $28(15,240)$ \\
\hline Attended a hospital or a clinic in the last 30 days & $16(17,588)$ & $5(1,594)$ & $5(2,015)$ & $26(21,197)$ \\
\hline Received chemotherapy in the last 30 days & $15(10,883)$ & $6(2,506)$ & $7(3,043)$ & $28(16,432)$ \\
\hline Receiving hemodialysis & $23(31,272)$ & $7(2,639)$ & $10(10,470)$ & $40(44,381)$ \\
\hline Prior hospitalization (in the last year) & $24(32,165)$ & $7(3,239)$ & $12(13,904)$ & $43(49,308)$ \\
\hline Resident in a nursing home or long term-care facility & $23(25,468)$ & $7(3,239)$ & $11(9,361)$ & $41(38,068)$ \\
\hline \multicolumn{5}{|l|}{ Criteria NOT included in the initial definition [3] } \\
\hline Transfer from another care facility & $1(6,697)$ & & $1(4,543)$ & $2(11,240)$ \\
\hline Immunosuppression & $4(8,669)$ & & & $4(8,669)$ \\
\hline Active or metastatic cancer & $1(7,712)$ & & & $1(7,712)$ \\
\hline Submitted to invasive procedures previously & $1(319)$ & $1(912)$ & $1(831)$ & $3(2,062)$ \\
\hline Family member with a multi-drug resistant microorganism & $2(713)$ & & $2(1,545)$ & $4(2,258)$ \\
\hline Elderly person or physical disable persons who need healthcare & $1(893)$ & & $1(1,385)$ & $2(2,278)$ \\
\hline Surgery in the last 180 days & $2(1,056)$ & & & $2(1,056)$ \\
\hline Received radiation therapy & $1(1,646)$ & & & $1(1,646)$ \\
\hline Recent (30 days) treatment with antibiotics & $1(190)$ & $1(733)$ & $1(197)$ & $3(1,120)$ \\
\hline
\end{tabular}

HCAl, healthcare-associated infection. 
The following criteria that were used in various studies to define HCAI in patients with an infection present at hospital admission or within 48 hours of admission are the ones that we believe to be most important:

- received invasive procedures in the 30 days before the infection, including specialized nursing care;

- attended a hospital or hemodialysis clinic in the previous 30 days;

- were hospitalized in an acute care hospital for 2 or more days in the previous year;

- resided in a nursing home or long-term care facility;

- treatment with broad spectrum antibiotics in the last 30 days.

The initial HCAI definition [3] included treatments delivered at home or in an outpatient clinic and these criteria have been widely adopted in other studies. The receipt of intravenous therapy [7,14,30,31,35-39,41,45-47], wound care or specialized nursing care [34-37,41,42,45], and hemodialysis [7,11-14,31-42,44-46], as well as attendance at a hospital or clinic $[32,42,44,45]$ are important factors as this group of patients has documented higher rates of colonization and infection with MDR microorganisms [48-50]. Three additional studies have included the criteria of other previous invasive procedures $[7,30,45]$, like urological procedures [45]. There is no reason to believe that this last group of patients is different from the previous ones in regards to the risks of infection by MDR organisms, so we propose that the first criterion be generalized to include all patients that received invasive procedures in the 30 days before the infection.

The second criterion in the initial definition [3] includes receiving chemotherapy in the last 30 days. This is a criterion frequently used among alternative definitions $[7,14,30,31,35,38-40,42,44,45]$ along with having active or metastatic cancer $[11,32]$ that suggest receipt of some kind of anti-cancer therapy. These are a special group of patients due to underlying immunosuppression. Immunosuppression, including HIV infection and treatment with immunosuppressive agents is a criterion considered by some authors $[11,12,33,34,38,39]$, but specifically excluded by others $[40,44]$. The variety of potential opportunistic pathogens that may occur among this group of patients varies largely according to the underlying cause of immunosuppression, for example empiric antimicrobial recommendations for a patient with advanced HIV infection [51] are distinct from therapies used in patients with acute febrile neutropenia [52]. The inclusion of these groups of patients in a HCAI definition is possibly one of the most controversial issues and for the moment we suggest that they be excluded from the definition, supported by the existence of specific recommendations for these special populations.
Nevertheless, many immunosuppressed patients, including cancer patients would fulfill other criteria for HCAI, such as invasive procedures, recently attending a hospital clinic, recent hospitalization and/or recent treatment with broad spectrum antibiotics.

Regarding previous hospitalization, we believe that this criterion must be retained in any definition of HCAI. The presence of MDR organisms (gram positives or gram negatives) has been documented between six months to one year after hospital discharge [53-55]. This risk of long lasting colonization of both the respiratory tract and gastrointestinal tract with pathogens not present in the community following hospitalization has led some authors to alter this criterion to hospitalization in the previous six months $[12,42,44]$ or even one year [33]. However, the classification of infections that develop among patients recently discharged from the hospital (in the previous 14 days) is somewhat contentious. Some authors consider these infections occurring within 14 days of hospital discharge nosocomial infections $[48,50,56]$, while others consider infections among those hospitalized in the last month as HCAIs [11,14]. Based on the existing evidence, we propose that in the third criterion time from the last hospitalization will be enlarged to one year and patients discharged from the hospital within the last two weeks be considered as having a hospital-acquired infection.

Patients admitted from nursing homes with infection have been extensively studied and may constitute more than $50 \%$ of cases of healthcare-associated pneumonia [49]. This criterion has been considered by almost all studies; however, caution is needed with this approach. Patients with non-severe nursing home-acquired pneumonia (NHAP) have a pathogen distribution similar to those expected in CAP [57]. Among patients with severe NHAP, with organ dysfunction, resistant pathogens have been seen $[10,36,57]$. Poor functional status and increased age have been linked to an increased risk of infection with a MDR pathogen among NHAP patients [20,58], and are linked to the level of care provided in these facilities. Nursing homes with hospital-like wards carry the same infection risk by resistant pathogens as hospitals, and should best be considered as the analogous to HAIs. Clinicians should consider factors such as functional status and level of care required in selecting treatments for patients who reside in nursing homes.

Recent treatment with broad spectrum antibiotics has been identified as a risk factor for infection or colonization by MDR pathogens [59] and should also be considered both in the definition of HCAI and in selection of empiric antibiotics.

Patients with close contact with a family member with a MDR microorganism are part of the American Thoracic Society (ATS) definition of HCAP [2]. Currently, there are no epidemiological studies assessing the microbiological 
features of this particular group of patients [50] sustaining its inclusion on the HCAI definition.

Additional criteria not included in the initial definition [3] represent different descriptions of the same criteria: active or metastatic cancer, submission to invasive procedures or transfer from another care facility.

There has only been one previous review on HCAI to our knowledge. It concerns healthcare-associated pneumonia and is focused mainly on epidemiology [60]. The authors performed the search in PubMed, and included eight studies regardless of the definition used. No assessment of bias was made. A description of the definitions of HCAP used was not made. Five of those studies focused only on nursing-home acquired pneumonia. The remaining three studies of HCAP included by the authors were also included in the current analysis. Recently, new definitions of Lab-ID infections were published [61] based on laboratory testing data without a clinical evaluation of the patient, allowing colonization to be counted as infection. Nevertheless, this methodology might facilitate surveillance of multi-drug resistant organisms (MDROs) among patients in the outpatient clinic and long-term care facilities and nursing home settings. Of notice is the fact that the document categorizes MDRO LabID events in: community-onset if the specimen was collected as an outpatient or inpatient three or more days after admission and healthcare facility-onset if the LabID event specimen was collected more than three days after admission to the facility. Following this definition, a patient with HCAI is included in community-onset LabID event, representing from our point of view a major step backward in the classification of infection according to place of acquisition.

This systematic review provides the clinician with a thorough description of all criteria available in order to include an infected patient in the category of HCAI, in the hope that it leads to an optimal selection of empiric antibiotic therapy in this group of patients and consequently an improvement in outcome. It is expected that a consensus definition of HCAI can be developed to be used in future research in order to develop specific antibiotic recommendations for this group of patients.

The future definition of HCAI should be universal regardless of the focus of infection if its use is intended to be immediate at the bedside like it happened with the classic dichotomy classification of infection in community and hospital-acquired infection, which allowed the prompt institution of adequate antibiotic therapy, a major prognostic factor. Nevertheless, specific risk factors for infection by a particular microorganism should always be taken into account by the clinician.

\section{Strengths and limitations}

Despite the extensive research done, including electronic search in several databases, relevant conference proceedings and a hand search of additional sources, there is always the possibility of missing studies that could meet the inclusion criteria.

Researcher bias is always a possibility in this type of analysis; in order to reduce it we had two independent researchers review the articles and a third one resolve disagreements along with strict and simple inclusion criteria established prior to the research.

The permissive criteria for inclusion in this study were essential to achieve the main goal: gathering all definitions of HCAI used in clinical studies.

We found a high rate of studies with low risk of bias, probably related to the simplicity of the evaluation. Considering that we only found observational studies we think that the criteria adopted were the most adequate to evaluate risk of bias in this type of studies.

\section{Conclusions and recommendations}

The initial definition of HCAI [3] seems to be widely accepted. Some of the included criteria, such as attendance at a hospital or hemodialysis clinic in the previous 30 days and residence in a nursing home or long-term care facility should be maintained; the precise time from the last hospitalization is still controversial and probably should be extended to one year. Additional criteria as recent invasive procedures and receipt of broad-spectrum antibiotics should be considered for inclusion in a future definition of HCAI.

The inclusion/exclusion of immunosuppressed patients in the definition of HCAI requires ongoing discussion.

It is expected that a consensus definition of HCAI can be developed soon to be used in future research in order to develop specific antibiotic recommendations for this group of patients, with an influence from local antibiograms.

\section{Additional files}

Additional file 1: Search strategy details.

Additional file 2: eTable 1. Studies with moderate or high risk of bias according to pre-defined criteria.

\section{Abbreviations}

ATS: American Thoracic Society; CAl: Community- acquired infection; CAP: Community-acquired pneumonia; ESBL: Extended-spectrum beta lactamases producer; HAl: Hospital-acquired infection; HAP: Hospital acquired pneumonia; HCAl: Healthcare-associated infection; HCAP: Healthcare-associated pneumonia; HIV: Human immunodeficiency virus; MDR: Multidrug-resistant; MDRO: multi-drug resistant organism; MRSA: Methicillin-resistant

Staphylococcus aureus; NHAP: Nursing home-acquired pneumonia.

\section{Competing interests}

The authors declare that they do not have any competing interests. 


\section{Authors' contributions}

TC, MA, LA, IA, ACP and AS were responsible for study concept and design. $\mathrm{TC}$ and MA acquired the data. TC, MA, LA and NDF were responsible for analysis and interpretation of data and drafting of the manuscript. All the authors took part in the revision of the manuscript and approval of the final version to be published.

\section{Acknowledgements}

This research was supported by an ASSUCIP (Associação de Apoio à Unidade de Cuidados Intensivos Polivalente - ICU, Hospital de Santo António, Porto, Portugal) grant.

Dr. Cardoso is partially funded by a PhD research grant from the Teaching and Research Department (Departamento de Formação, Ensino e Investigação) of Oporto Hospital Centre.

The funding organization had no role in the design or conduct of the study; the collection, analysis or interpretation of the data; or the preparation, review or approval of the manuscript, nor in the decision to submit the manuscript for publication.

\section{Author details}

${ }^{1}$ Intensive Care Unit, Unidade de Cuidados Intensivos Polivalente, Hospital de Santo António, University of Porto, Largo Prof. Abel Salazar, 4099-001 Porto, Portugal. ${ }^{2}$ Internal Medicine Department, Hospital de Braga, Sete Fontes São Vitor, 4710-243 Braga, Portugal. ³ Department of Medicine, Barwon Health, Geelong, VIC 3220, Australia. ${ }^{4}$ Department of Health Information and Decision Sciences, Center for Research in Health Technologies and Information Systems (CINTESIS), Faculty of Medicine, University of Porto, Alameda Prof. Hernâni Monteiro, 4200-319 Porto, Portugal. ${ }^{5}$ Department of Infectious Diseases, Hospital de São João, University of Porto, Alameda Prof. Hernâni Monteiro, 4200-319 Porto, Portugal.

\section{Received: 27 November 2013 Accepted: 11 February 2014} Published: 6 March 2014

\section{References}

1. Garner JS, Jarvis WR, Emori TG, Horan TC, Hughes JM: CDC definitions for nosocomial infections, 1988. Am J Infect Control 1988, 16:128-140.

2. American Thoracic Society; Infectious Diseases Society of America: Guidelines for the management, of adults with hospital-acquired, ventilator-associated, and healthcare-associated pneumonia. Am J Respir Crit Care Med 2005, 171:388-416.

3. Friedman ND, Kaye KS, Stout JE, McGarry SA, Trivette SL, Briggs JP, Lamm W, Clark C, MacFarquhar J, Walton AL, Reller LB, Sexton DJ: Health care-associated bloodstream infections in adults: a reason to change the accepted definition of community-acquired infections. Ann Intern Med 2002, 137:791-797.

4. Park SC, Kang YA, Park BH, Kim EY, Park MS, Kim YS, Kim SK, Chang J, Jung JY: Poor prediction of potentially drug-resistant pathogens using current criteria of health care-associated pneumonia. Respir Med 2012, 106:1311-1319.

5. Benito N, Miró JM, de Lazzari E, Cabell CH, del Río A, Altclas J, Commerford P, Delahaye F, Dragulescu S, Giamarellou H, Habib G, Kamarulzaman A, Kumar AS, Nacinovich FM, Suter F, Tribouilloy C, Venugopal K, Moreno A, Fowler VG Jr, ICE-PCS (International Collaboration on Endocarditis Prospective Cohort Study) Investigators: Health care-associated native valve endocarditis: importance of non-nosocomial acquisition. Ann Intern Med 2009, 150:586-594. Erratum in: Ann Intern Med 2010, 152:480.

6. Aguilar-Duran S, Horcajada JP, Sorlí L, Montero M, Salvadó M, Grau S, Gómez J, Knobel H: Community-onset healthcare-related urinary tract infections: comparison with community and hospital-acquired urinary tract infections. J Infect 2012, 64:478-483.

7. Siegman-Igra Y, Fourer B, Orni-Wasserlauf R, Golan Y, Noy A, Schwartz D, Giladi M: Reappraisal of community-acquired bacteremia: a proposal of a new classification for the spectrum of acquisition of bacteremia. Clin Infect Dis 2002, 34:1431-1439.

8. Vallés J, Alvarez-Lerma F, Palomar M, Blanco A, Escoresca A, Armestar F, Sirvent JM, Balasini C, Zaragoza R, Marín M, Study Group of Infectious Diseases of the Spanish Society of Critical Care Medicine: Health-care-associated bloodstream infections at admission to the ICU. Chest 2011, 139:810-815.

9. Park HK, Song J-U, Um S-W, Koh W-J, Suh GY, Chung MP, Kim H, Kwon OJ, Jeon K: Clinical characteristics of health care-associated pneumonia in a Korean teaching hospital. Respir Med 2010, 104:1729-1735.
10. Carratalà J, Mykietiuk A, Fernández-Sabé N, Suárez C, Dorca J, Verdaguer R, Manresa F, Gudiol F: Health care-associated pneumonia requiring hospital admission: epidemiology, antibiotic therapy, and clinical outcomes. Arch Intern Med 2007, 167:1393-1399.

11. Shorr AF, Tabak YP, Killian AD, Gupta V, Liu LZ, Kollef MH: Healthcare-associated bloodstream infection: a distinct entity? Insights from a large US database. Crit Care Med 2006, 34:2588-2595.

12. Kollef MH, Zilberberg MD, Shorr AF, Vo L, Schein J, Micek ST, Kim M: Epidemiology, microbiology and outcomes of healthcare-associated and community-acquired bacteremia: a multicenter cohort study. J Infect 2011, 62:130-135.

13. Kollef MH, Shorr A, Tabak YP, Gupta V, Liu LZ, Johannes RS: Epidemiology and outcomes of healthcare-associated pneumonia: results from a large US database of culture-positive pneumonia. Chest 2005, 128:3854-3862. Erratum in Chest 2006, 129:831.

14. Grenier C, Pepin J, Nault V, Howson J, Fournier X, Poirier M-S, Cabana J, Craig C, Beaudoin M, Valiquette L: Impact of guideline-consistent therapy on outcome of patients with healthcare-associated and community-acquired pneumonia. J Antimicrob Chemother 2011, 66:1617-1624.

15. Pop-Vicas AE, D'Agata EM: The rising influx of multidrug-resistant gramnegative bacilli into a tertiary care hospital. Clin Infect Dis 2005, 40:1792-1798.

16. Marschall J, Fraser VJ, Doherty J, Warren DK: Between community and hospital: healthcare-associated gram-negative bacteremia among hospitalized patients. Infect Control Hosp Epidemiol 2009, 30:1050-1056.

17. Evans $C T$, Hershow RC, Chin A, Foulis PR, Burns SP, Weaver FM: Bloodstream infections and setting of onset in persons with spinal cord injury and disorder. Spinal Cord 2009, 47:610-615.

18. Son JS, Song J-H, Ko KS, Yeom JS, Ki HK, Kim S-W, Chang H-H, Ryu SY, Kim Y-S, Jung S-I, Shin SY, Oh HB, Lee YS, Chung DR, Lee NY, Peck KR: Bloodstream infections and clinical significance of healthcare-associated bacteremia: a multicenter surveillance study in Korean hospitals. J Korean Med Sci 2010, 25:992-998.

19. Rodríguez-Baño J, López-Prieto MD, Portillo MM, Retamar P, Natera C, Nuño E, Herrero M, del Arco A, Muñoz A, Téllez F, Torres-Tortosa M, Martín-Aspas A, Arroyo A, Ruiz A, Moya R, Corzo JE, León L, Pérez-López JA, SAEI/SAMPAC Bacteraemia Group: Epidemiology and clinical features of community-acquired, healthcare-associated and nosocomial bloodstream infections in tertiary-care and community hospitals. Clin Microbiol Infect 2010, 16:1408-1413.

20. Shindo Y, Sato S, Maruyama E, Ohashi T, Ogawa M, Hashimoto N, Imaizumi K, Sato T, Hasegawa Y: Health-care-associated pneumonia among hospitalized patients in a Japanese Community Hospital. Chest 2009, 135:633-640.

21. Pascual V, Salvador M, Calvo E, Freixas N, Riera M, Xercavins M, Garau J: Healthcare-associated pneumonia: a category under review. Abstr Intersci Conf Antimicrob Agents Chemother 2010, 50:P1658.

22. Umeki K, Tokimatsu I, Yasuda C, Iwata A, Yoshioka D, Ishii H, Shirai R, Kishi K, Hiramatsu K, Matsumoto B, Kadota J: Clinical features of healthcare-associated pneumonia (HCAP) in a Japanese community hospital: comparisons among nursing home-acquired pneumonia (NHAP), HCAP other than NHAP, and community-acquired pneumonia. Respirology 2011, 16:856-861.

23. Seki M, Hashiguchi K, Tanaka A, Kosai K, Kakugawa T, Awaya Y, Kurihara S, Izumikawa K, Kakeya H, Yamamoto Y, Yanagihara K, Tashiro T, Kohno S: Characteristics and disease severity of healthcare-associated pneumonia among patients in a hospital in Kitakyushu, Japan. J Infect Chemother 2011, 17:363-369.

24. Garcia-Vidal C, Viasus D, Roset A, Adamuz J, Verdaguer R, Dorca J, Gudiol F, Carratalà J: Low incidence of multidrug-resistant organisms in patients with healthcare-associated pneumonia requiring hospitalization. Clin Microbiol Infect 2011, 17:1659-1665.

25. Jung JY, Park MS, Kim YS, Park BH, Kim SK, Chang J, Kang YA: Healthcareassociated pneumonia among hospitalized patients in a Korean tertiary hospital. BMC Infect Dis 2011, 11:61. doi:10.1186/1471-2334-11-61.

26. Jeon EJ, Cho S-G, Shin JW, Kim JY, Park IW, Choi BW, Choi JC: The difference in clinical presentations between healthcare-associated and communityacquired pneumonia in university-affiliated hospital in Korea. Yonsei Med J 2011, 52:282-287.

27. Depuydt P, Putman B, Benoit D, Buylaert W, De Paepe P: Nursing home residence is the main risk factor for increased mortality in healthcare-associated pneumonia. J Hosp Infect 2011, 77:138-142.

28. Lee $\mathrm{JH}, \mathrm{Kim} \mathrm{YH}$ : Comparison of clinical characteristics between healthcareassociated pneumonia and community-acquired pneumonia in patients admitted to secondary hospitals. Braz J Infect Dis 2012, 16:321-328. 
29. Wu KS, Lee SS, Tsai HC, Wann SR, Chen JK, Sy CL, Wang YH, Tseng YT, Chen YS: Non-nosocomial healthcare-associated infective endocarditis in Taiwan: an underrecognized disease with poor outcome. BMC Infect Dis 2011, 11:221. doi:10.1186/1471-2334-11-221.

30. Kao CH, Kuo YC, Chen CC, Chang YT, Chen YS, Wann SR, Liu YC: Isolated pathogens and clinical outcomes of adult bacteremia in the emergency department: a retrospective study in a tertiary referral center. J Microbiol Immunol Infect 2011, 44:215-221.

31. Al-Hasan MN, Eckel-Passow JE, Baddour LM: Impact of healthcare-associated acquisition on community-onset Gram-negative bloodstream infection: a population-based study: healthcare-associated Gram-negative BSI. Eur J Clin Microbiol Infect Dis 2012, 31:1163-1171.

32. Lenz R, Leal JR, Church DL, Gregson DB, Ross T, Laupland KB: The distinct category of healthcare associated bloodstream infections. BMC Infect Dis 2012, 12:85. doi:10.1186/1471-2334-12-85.

33. Micek ST, Kollef KE, Reichley RM, Roubinian N, Kollef MH: Health care-associated pneumonia and community-acquired pneumonia: a single-center experience. Antimicrob Agents Chemother 2007, 51:3568-3573.

34. Schreiber MP, Chan CM, Shorr AF: Resistant pathogens in Nonnosocomial pneumonia and respiratory failure is it time to refine the definition of health-care-associated pneumonia? Chest 2010, 137:1283-1288.

35. Guimaraes C, Lares Santos C, Costa F, Barata F: Pneumonia associated with health care versus community acquired pneumonia: different entities, distinct approaches. Rev Port Pneumol 2011, 17:168-171.

36. Chalmers JD, Taylor JK, Singanayagam A, Fleming GB, Akram AR, Mandal P, Choudhury G, Hill AT: Epidemiology, antibiotic therapy, and clinical outcomes in health care-associated pneumonia: a UK cohort study. Clin Infect Dis 2011, 53:107-113.

37. Taşbakan MS, Bacakoğlu F, Kaçmaz Başoğlu O, Gürgün A, Başarik B, Çitim Tuncel S, Sayiner A: The comparison of patients with hospitalized healthcare-associated pneumonia to community-acquired pneumonia. Tüberküloz ve Toraks Dergisi 2011, 59:348-354.

38. Ishida T, Tachibana H, Ito A, Yoshioka H, Arita M, Hashimoto T: Clinical characteristics of nursing and healthcare-associated pneumonia: a Japanese variant of healthcare-associated pneumonia. Intern Med 2012, 51:2537-2544.

39. Miyashita N, Kawai Y, Akaike H, Ouchi K, Hayashi T, Kurihara T, Okimoto N: Clinical features and the role of atypical pathogens in nursing and healthcare-associated pneumonia (NHCAP): differences between a teaching university hospital and a community hospital. Intern Med 2012, 51:585-594

40. Wu CL, Ku SC, Yang KY, Fang WF, Tu CY, Chen CW, Hsu KH, Fan WC, Lin MC, Chen W, Ou CY, Yu CJ: Antimicrobial drug-resistant microbes associated with hospitalized community-acquired and health care-associated pneumonia: a multi-center study in Taiwan. J Formos Med Assoc 2013, 112:31-40.

41. Sugisaki M, Enomoto $T$, Shibuya $Y$, Matsumoto $A$, Saitoh $H$, Shingu A, Narato R, Nomura K: Clinical characteristics of healthcare-associated pneumonia in a public hospital in a metropolitan area of Japan. $J$ Infect Chemother 2012, 18:352-360.

42. Giannella M, Pinilla B, Capdevila JA, Martínez Alarcón J, Muñoz P, López Álvarez J, Bouza E, on behalf of the Estudio de Neumonía En Medicina Interna (ENEMI) study Group from the Sociedad Española de Medicina Interna (SEMI): Pneumonia treated in the internal medicine department: focus on healthcare-associated pneumonia. Clin Microbiol Infect 2012, 18:786-794.

43. Swenson BR, Metzger R, Hedrick TL, McElearney ST, Evans HL, Smith RL, Chong TW, Popovsky KA, Pruett TL, Sawyer RG: Choosing antibiotics for intra-abdominal infections: what do we mean by "high risk"? Surg Infect 2009, 10:29-39.

44. Merli M, Lucidi C, Giannelli V, Giusto M, Riggio O, Falcone M, Ridola L, Attili $A F$, Venditti M: Cirrhotic patients are at risk for health care-associated bacterial infections. Clin Gastroenterol Hepatol 2010, 8:979-985.

45. Ha YE, Kang C-I, Joo E-J, Park SY, Kang SJ, Wi YM, Chung DR, Peck KR, Lee $\mathrm{NY}$, Song J-H: Clinical implications of healthcare-associated infection in patients with community-onset acute pyelonephritis. Scand J Infect Dis 2011, 43:587-595.

46. Sy RW, Kritharides L: Health care exposure and age in infective endocarditis: results of a contemporary population-based profile of 1536 patients in Australia. Eur Heart J 1890-1897, 2010:31.

47. Liu X, Han X, Ge Y, Wei F: Clinical analysis of healthcare-associated pneumonia. Chin J Infect Chemother 2011, 11:452-456.
48. Falcone $M$, Venditti $M$, Shindo $Y$, Kollef MH: Healthcare-associated pneumonia: diagnostic criteria and distinction from community-acquired pneumonia. Int J Infect Dis 2011, 15:E545-E550.

49. Attridge RT, Frei CR: Health care-associated pneumonia: an evidence-based review. Am J Med 2011, 124:689-697.

50. Lopez A, Amaro R, Polverino E: Does health care associated pneumonia really exist? Eur J Intern Med 2012, 23:407-411.

51. Seddon J, Bhagani S: Antimicrobial therapy for the treatment of opportunistic infections in HIV/AIDS patients: a critical appraisal. HIV Aids 2011, 3:19-33.

52. Donowitz GR, Maki DG, Crnich CJ, Pappas PG, Rolston KV: Infections in the neutropenic patient-new views of an old problem. Hematology Am SoC Hematol Educ Program 2001:113-139. doi:10.1182/asheducation-2001.1.113.

53. Naimi TS, LeDell KH, Como-Sabetti K, Borchardt SM, Boxrud DJ, Etienne J, Johnson SK, Vandenesch F, Fridkin S, O'Boyle C, Danila RN, Lynfield R: Comparison of community- and health care-associated methicillin-resistant Staphylococcus aureus infection. JAMA 2003, 290:2976-2984.

54. Zahar JR, Lanternier F, Mechai F, Filley F, Taieb F, Mainot EL, Descamps P, Corriol O, Ferroni A, Bille E, Nassif X, Lortholary O: Duration of colonisation by Enterobacteriaceae producing extended-spectrum beta-lactamase and risk factors for persistent faecal carriage. J Hosp Infect 2010, 75:76-78.

55. Chen SY, Wu GH, Chang SC, Hsueh PR, Chiang WC, Lee CC, Ma MH, Hung CC, Chen YC, Su CP, Tsai KC, Chen TH, Chen SC, Chen WJ: Bacteremia in previously hospitalized patients: prolonged effect from previous hospitalization and risk factors for antimicrobial-resistant bacterial infections. Ann Emerg Med 2008, 51:639-646.

56. Collins AS: Preventing health care-associated infections. In Patient Safety and Quality: an Evidence-Based Handbook for Nurses. Edited by Hughes RG. Rockville (MD): Agency for Healthcare Research and Quality, U.S. Department of Health and Human Services; 2008.

57. Polverino E, Dambrava P, Cilloniz C, Balasso V, Marcos MA, Esquinas C, Mensa J, Ewig S, Torres A: Nursing home-acquired pneumonia: a 10 year single-centre experience. Thorax 2010, 65:354-359.

58. El Solh AA, Pietrantoni C, Bhat A, Bhora M, Berbary E: Indicators of potentially drug-resistant bacteria in severe nursing home-acquired pneumonia. Clin Infect Dis 2004, 39:474-480.

59. Mandell LA, Wunderink RG, Anzueto A, Bartlett JG, Campbell GD, Dean NC, Dowell SF, File TM Jr, Musher DM, Niederman MS, Torres A, Whitney CG, Infectious Diseases Society of America; American Thoracic Society: Infectious Diseases Society of America/American Thoracic Society consensus guidelines on the management of community-acquired pneumonia in adults. Clin Infect Dis 2007, 44:S27-S72.

60. Brito V, Niederman MS: Healthcare-associated pneumonia is a heterogeneous disease, and all patients do not need the same broad-spectrum antibiotic therapy as complex nosocomial pneumonia. Curr Opin Infect Dis 2009, 22:316-325.

61. Centers for Disease Control and Prevention: Multidrug resistant organism \& Clostridium difficile infection (MDRO/CDI) module. [http://www.cdc. gov/nhsn/PDFs/pscManual/12pscMDRO_CDADcurrent.pdf]

doi:10.1186/1741-7015-12-40

Cite this article as: Cardoso et al:: Classification of healthcare-associated infection: a systematic review 10 years after the first proposal. BMC Medicine 2014 12:40.

\section{Submit your next manuscript to BioMed Central and take full advantage of:}

- Convenient online submission

- Thorough peer review

- No space constraints or color figure charges

- Immediate publication on acceptance

- Inclusion in PubMed, CAS, Scopus and Google Scholar

- Research which is freely available for redistribution 